\title{
Methylation of the tryptophan hydroxylase-2 gene is associated with mRNA expression in patients with major depression with suicide attempts
}

\author{
YUQI ZHANG ${ }^{1}$, ZAOHUO CHANG $^{1}$, JIONGHUA CHEN $^{2}$, YANG LING $^{3}$, XIAOWEI LIU ${ }^{1}$, \\ ZHANG FENG $^{1}$, CAIXIA CHEN ${ }^{1}$, MINGHUA XIA ${ }^{1}$, XINGFU ZHAO ${ }^{1}$, \\ WANG YING ${ }^{1}, \mathrm{XU}$ QING $^{1}$, GUILIN $\mathrm{LI}^{1}$ and CHANGSONG ZHANG ${ }^{3}$ \\ ${ }^{1}$ Department of Geriatric Psychiatry, Wuxi Psychiatric Hospital, Nanjing Medical University, Wuxi, Jiangsu 214151; \\ ${ }^{2}$ Department of Brain, Jiangsu Province Academy of Traditional Chinese Medicine, Nanjing, Jiangsu 210028; \\ ${ }^{3}$ Clinical Oncology Laboratory, Changzhou Cancer Hospital of Soochow University, Changzhou, Jiangsu 213002, P.R. China
}

Received July 24, 2014; Accepted April 9, 2015

DOI: $10.3892 / \mathrm{mmr} .2015 .3748$

\begin{abstract}
Tryptophan hydroxylase-2 (TPH2) contributes to alterations in the function of neuronal serotonin (5-HT), which are associated with various psychopathologies, including major depressive disorder (MDD) or suicidal behavior. The methylation of a single $\mathrm{CpG}$ site in the promoter region of TPH2 affects gene expression. Suicide and MDD are strongly associated and genetic factors are at least partially responsible for the variability in suicide risk. The aim of the present study was to investigate whether variations in TPH2 methylation in peripheral blood samples may predispose patients with MDD to suicide attempts. TPH2 mRNA expression levels differed significantly between 50 patients with MDD who had attempted suicide (MDD + suicide group) and 75 control patients with MDD (MDD group); TPH2 expression levels were significantly decreased $(\mathrm{P}=0.0005)$ in the patients who had attempted suicide. Furthermore, the frequency of TPH2 methylation was $36.0 \%$ in the MDD + suicide group, while it was $13.0 \%$ in the MDD group. The results of the present study demonstrated that methylation in the promoter region of TPH2 significantly affected the mRNA expression levels of $\mathrm{TPH} 2$, thus suggesting that methylation of the TPH2 promoter may silence TPH2 mRNA expression in MDD patients with or without suicidal behavior. In addition, there was a
\end{abstract}

Correspondence to: Dr Changsong Zhang, Clinical Oncology Laboratory, Changzhou Cancer Hospital of Soochow University, 1 North Huaide Road, Changzhou, Jiangsu 213002, P.R. China E-mail: hbzcs@126.com

Dr Yuqi Zhang, Department of Geriatric Psychiatry, Wuxi Psychiatric Hospital, Nanjing Medical University, 156 Qianrong Road, Wuxi, Jiangsu 214151, P.R. China

E-mail: g_yqzhang@126.com

Key words: major depression disorders, suicide attempt, tryptophan hydroxylase-2, methylation significant correlation between the methylation status of the TPH2 promoter and depression, hopelessness and cognitive impairment in the MDD + suicide group. In conclusion, the present study demonstrated that TPH 2 expression was regulated by DNA methylation of the TPH 2 promoter region in patients with MDD.

\section{Introduction}

Suicide is a major public health problem that has received increasing attention worldwide (1). In the majority of studies regarding death by suicide, $\sim 9$ out of every 10 individuals appear to have had a psychiatric disorder at the time of their death (2). Depression is the most common psychiatric disorder in people who commit suicide (3). Depression is strongly associated with suicide and non-fatal suicidal behaviors, and requires an ongoing assessment due to suicide risk. Patients with major affective disorders, such as major depressive disorder (MDD), are highly vulnerable to suicidal behavior (4).

Serotonin (5-HT) is a monoamine implicated in various physiological processes, which functions as a neurotransmitter and a peripheral hormone. 5-HT is associated with the pathophysiology of depressive disorders. The peripheral and cerebral biosynthesis of 5-HT is initiated by two distinct tryptophan hydroxylases (TPH): TPH1 and TPH2 (5). TPH1 is predominantly expressed in the periphery and the pineal gland, whereas TPH2 is thought to be neuron-specific and is predominantly expressed in the brain; however, it may also be expressed or induced to be expressed in peripheral tissues (6). Of note, numerous studies have indicated that TPH2 gene expression is highly inducible and is closely associated with depressive disorders $(7,8)$.

Previous studies have linked TPH2 genetic variance to various behavioral traits and disorders (9-11). It has been hypothesized that functional polymorphisms involving the promoter region of $\mathrm{TPH} 2$, which affect gene expression, may explain this finding $(12,13)$. The presence of a functional cis-acting polymorphism, with high frequency in normal human subjects, results in increased expression levels of TPH2, 
and has been shown to be associated with major depression and suicide (14). A study demonstrated that the 5'-untranslated region (UTR) and common polymorphisms in the 5 ' regulatory region of human TPH2 have a significant impact on gene expression (15). Non-synonymous single nucleotide polymorphisms (SNPs) in rhesus monkey TPH2 have been shown to affect mRNA stability, thus suggesting that non-synonymous SNPs affect TPH2 function and gene expression (16). The TPH2 SNPs rs1386482 and rs1386486 have previously been associated with bipolar affective disorder (17). In addition, the rs4570625 SNP of TPH2 were suggested to have an important role in the development of positive symptoms in Han Chinese patients with schizophrenia (18). A previous study by our group indicated that TPH2 rs7305115A remained a significant protective predictor of suicide attempts (19). However, little is currently known regarding the impact of TPH 2 gene variants on expression $(20,21)$.

Recent evidence regarding DNA methylation alterations within distinct genes and pathways provided novel insight into the pathophysiology of psychiatric disorders $(22,23)$. DNA methylation, even in peripheral tissues, appears to be an informative reflection of environmental exposure of the genome, and may have potential as a biomarker for the early prevention of depressive disorders (24). The TPH2 promoter contains no $\mathrm{CpG}$ island; however, it does contain numerous scattered $\mathrm{CpG}$ sites and an enriched signal of DNA hypomethylation at the 5'-UTR locus (6). To identify the molecular basis of gene expression variation, the present study conducted association studies between TPH 2 promoter methylation and TPH 2 transcription levels in 50 patients with MDD who had attempted suicide and 75 control subjects.

\section{Patients and methods}

Patients and controls. The subjects of the present study consisted of 125 unrelated patients with MDD, who were recruited from the Han population of Jiangsu (China) between 2010 and 2013. All patients (58 males, 67 females) were hospitalized in the Department of Geriatric Psychiatry, Wuxi Psychiatric Hospital (Wuxi, China). Diagnosis of MDD was confirmed using the Mini International Neuropsychiatric Interview, and by a minimum score of Hamilton Depression Rating Scale (HDRS) $(25,26)$. All cases met the DSM-IV diagnostic criteria for MDD and were severe enough to require follow-up in a specialized psychiatric outpatient clinic at the Department of Geriatric Psychiatry, Wuxi Psychiatric Hospital (27,28). The HAMA scale was used to assess the severity of symptoms (29) by means of structured questionnaires, information on specific demographic and clinical variables, including family history of suicide was obtained.

A total of $50 \mathrm{MDD}+$ suicide patients (defined as the MDD + suicide attempts group; 23 males, and 27 females) who were consecutively admitted to our psychiatric departments following a suicide attempt, were included in the present study. Suicide was defined as intentional self-harm, in order to end one's life. The remaining $75 \mathrm{MDD}$ control patients (defined as the MDD group; 35 males, and 40 females) who had not attempted suicide were selected for the present study. Written informed consent was obtained from each patient, and the protocol of the study was approved by the local ethics committee of Nanjing Medical University (Nanjing, China).

Bisulfite modification and methylation-specific polymerase chain reaction (MSP). Venous blood was collected from the patients and immediately frozen in aliquots at $-80^{\circ} \mathrm{C}$ or below, prior to analysis. For genotyping, genomic DNA was extracted from EDTA-supplemented blood samples using a commercial DNA extract kit (Wizard ${ }^{\circledR}$ Genomic DNA Purification kit; Promega Corp., Madison, WI, USA).

Genomic DNA (3 $\mu \mathrm{g})$ from the cells was denatured with $0.3 \mathrm{M} \mathrm{NaOH}$ (Sigma-Aldrich, Shanghai, China) at $37^{\circ} \mathrm{C}$ for $10 \mathrm{~min}$. Bisulfite treatment (Sigma-Aldrich) was performed as described previously (30). Primers specific for TPH2 MSP were designed using MethPrimer (31) and synthesized by Sangon Biotech Co. (Shanghai, China) . Bisulfite-treated DNA was used for amplification of the TPH2 promoter. Primers specific for TPH2 were as follows: Unmethylated (U) TPH2 sense, 5'-TTTGTAATTTGATTGTGGTTATTGG-3' and anti-sense, 5'-ACAATCAACTACCTACTTAAAACACT-3'; and methylated (M) TPH2 sense, 5'-GGTTTGTAATTTGAT TGTGGTTATC-3' and anti-sense, 5'-CGATCAACTACCTAC TTAAAACGCT-3', which amplify a 164-bp product and a 165-bp product, respectively. MSP consisted of 38 cycles at $95^{\circ} \mathrm{C}$ for $45 \mathrm{sec}, 60^{\circ} \mathrm{C}$ for $50 \mathrm{sec}$ and $72^{\circ} \mathrm{C}$ for $30 \mathrm{sec}$, followed by a 10 -min extension at $72^{\circ} \mathrm{C}$ in a DNA Thermocycler (Agilent Technologies, Inc., Santa Clara, CA, USA). The amplification products were separated by $2 \%$ agarose gel electrophoresis (Sangon Biotech Co.) and visualized by ethidium bromide staining (Sangon Biotech Co.) and ultraviolet transillumination (FR-2000 UV transillumination; Shanghai Furi Science \& Technology Co., Ltd., Shanghai, China). Methylation was defined as $\mathrm{M} /(\mathrm{M}+\mathrm{U}) \geq 0.5$; and unmethylation was defined as $\mathrm{M} /(\mathrm{M}+\mathrm{U})<0.5$.

Reverse-transcription quantitative polymerase chain reaction $(R T-q P C R)$. RT-qPCR was performed using SYBR Green I chemistry (Invitrogen Life Technologies, Carlsbad, CA, USA). Specific primers were designed to target human TPH2 (PrimerBank ID: 169234956c2; 130 bp). Total RNA was isolated from the blood samples. The first-strand cDNA was synthesized from a moloney murine leukemia virus-reverse transcriptase kit using $2 \mu \mathrm{g}$ total RNA according to the manufacturer's instructions (Takara Bio, Inc., Dalian, China). Primer sequences of TPH2 for the RT-qPCR reaction were as follows: Forward, 5'-CAAAAATGACGACAA AGGCAACA-3' and reverse, 5'-CCTCAGTGCTTTTAC CAATCCA-3'. Primer sequences of $\beta$-actin for the RT-qPCR reaction were as follows: Forward, 5'-CTGGGACGAATG GAGAAA-3' and reverse, 5'-AAGGAAGGCTGGAAG AGTGC-3'. The qPCR was performed using the Mx3000P qPCR system (Stratagene, La Jolla, CA, USA) and the PCR reaction mixture consisted of cDNA in $20 \mu \mathrm{l}$ SYBR Premix Ex Taq. The qPCR was performed under the following conditions: $5 \mathrm{~min}$ at $95^{\circ} \mathrm{C}$, followed by 40 cycles of $30 \mathrm{sec}$ at $95^{\circ} \mathrm{C}$, $30 \mathrm{sec}$ at $58^{\circ} \mathrm{C}$ and $50 \mathrm{sec}$ at $72^{\circ} \mathrm{C}$. As an internal control for qPCR, $\beta$-actin mRNA expression was amplified from the same cDNA samples. All of the results were normalized to $\beta$-actin. Cycle threshold $(\mathrm{Ct})$ values for triplicate reactions were averaged and relative TPH 2 expression levels were 
Table I. Clinical characteristics of patients with MDD, with or without suicidal behavior.

\begin{tabular}{|c|c|c|c|}
\hline Characteristic & MDD + suicide & MDD & P-value \\
\hline Gender (male/female) & $23 / 27$ & $35 / 40$ & 0.554 \\
\hline Age, range (mean $\pm \mathrm{SD}$ ) in years & $14-71(36.8 \pm 10.2)$ & $13-70(35.3 \pm 11.0)$ & 0.645 \\
\hline Age at onset, range (mean \pm SD) in years & $11-60(25.2 \pm 11.5)$ & $10-56(26.6 \pm 12.1)$ & 0.843 \\
\hline Course of disease, range $($ mean $\pm \mathrm{SD})$ & 2 weeks- 24 years $(3.73 \pm 3.82$ years $)$ & 2 weeks-26 years $(3.69 \pm 4.04$ years $)$ & 0.082 \\
\hline HDRS scores & $47.32 \pm 9.31$ & $48.65 \pm 9.14$ & \\
\hline
\end{tabular}

MDD, major depressive disorder; SD, standard deviation; HDRS, Hamilton Depression Rating Scale.

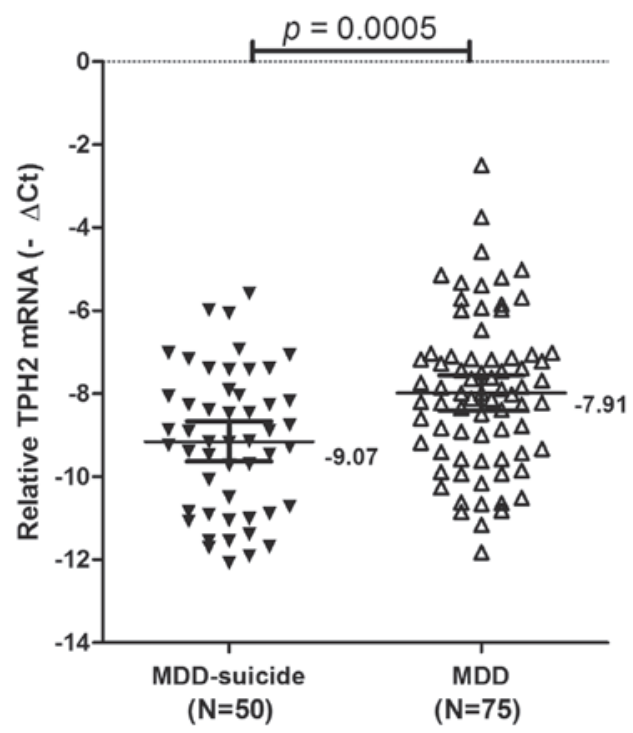

Figure 1. Relative mRNA expression levels of TPH2 in peripheral blood samples from the MDD + suicide attempts group and the MDD control group. Values are expressed as the mean $-\Delta \mathrm{Ct} \pm$ standard error. TPH 2 mRNA expression levels were lower in the MDD + suicide group, as compared with those in the MDD control group ( $\mathrm{P}=0.0005)$. Statistical analyses were conducted using an unpaired t-test. TPH2, tryptophan hydroxylase-2; MDD, major depressive disorder; $\mathrm{Ct}$ cycle threshold; $-\Delta \mathrm{Ct},-\left(\mathrm{Ct}_{\mathrm{TPH} 2}-\mathrm{Ct}_{\beta \text {-actin }}\right)$. Data are expressed as the mean \pm standard deviation.

determined using the comparative CT method (32), using the average $\mathrm{Ct}$ values for TPH 2 and $\beta$-actin.

Statistical analysis. All data were generated without knowledge of the clinical status of the samples, and were analyzed using SPSS 17.0 software (SPSS, Inc., Chicago, IL, USA). Differences between the groups were calculated using the unpaired t-test. Associations between categorical variables were examined using Pearson's $\chi^{2}$ and Fisher's exact tests. Data are expressed as the mean \pm standard deviation. $\mathrm{P}<0.05$ was considered to indicate a statistically significant difference.

\section{Results}

Clinical characteristics of patients. Demographic and clinical characteristics of the subjects of the present study are presented in Table I. The study population consisted of $50 \mathrm{MDD}+$ suicide patients [mean age \pm standard deviation

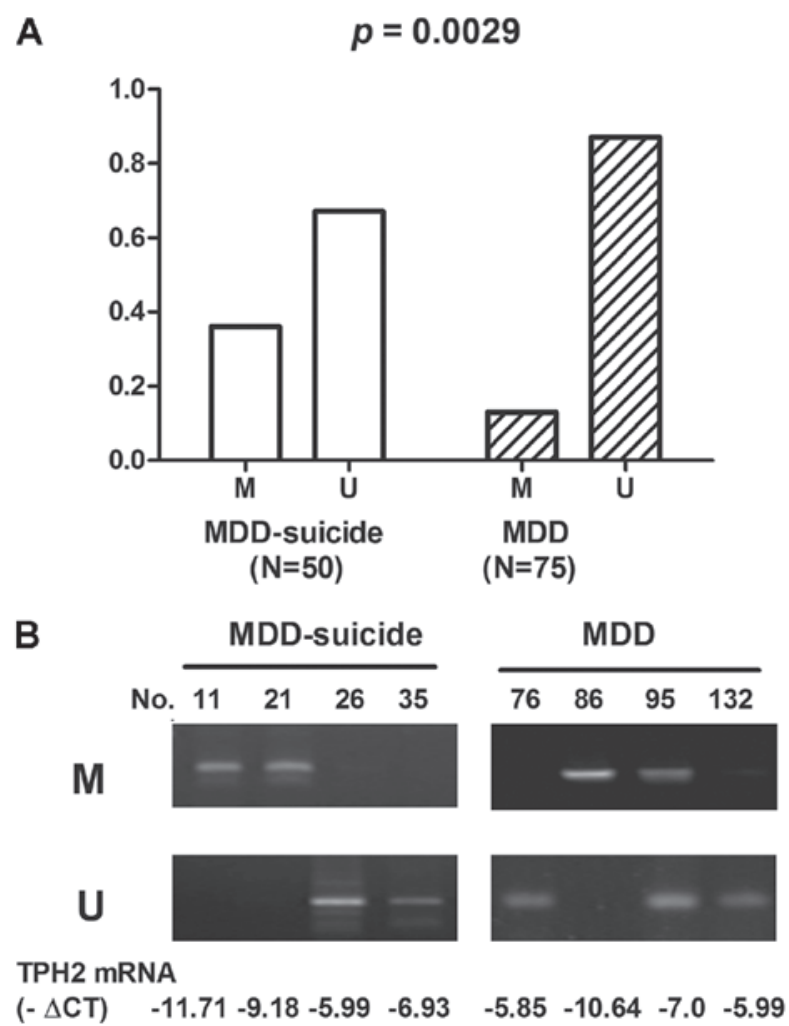

Figure 2. Promoter methylation of TPH2 in the MDD + suicide attempts and MDD control groups, as determined by methylation-specific polymerase chain reaction. (A) Methylation frequency of the TPH2 promoter in the MDD + suicide and MDD control groups. M, methylated; U, unmethylated; (B) Representative patterns of TPH2 promoter methylation. U, reaction specific for unmethylated DNA; M, reaction specific for methylated DNA. $-\Delta \mathrm{Ct}$, - $\left(\mathrm{Ct}_{\mathrm{TPH} 2}-\mathrm{Ct}_{\beta \text {-actin }}\right) ; \mathrm{TPH} 2$, tryptophan hydroxylase-2; MDD, major depressive disorder; $\mathrm{Ct}$, cycle threshold. Data are expressed as the mean \pm standard deviation.

(SD), $36.8 \pm 10.2$ years] and 75 matched MDD patients (mean age $\pm \mathrm{SD}, 35.3 \pm 11.0$ years). For the MDD + suicide group, 22 patients attempted suicide by drug overdose, hanging and drowning, and 28 patients attempted suicide by violent methods, such as through several deep cuts. No statistically significant differences were observed between the MDD + suicide and MDD groups for age, age at onset, course of disease or HDRS scores.

mRNA expression levels of TPH2 in the MDD + suicide and $M D D$ groups. To accurately quantify the relative mRNA 
A

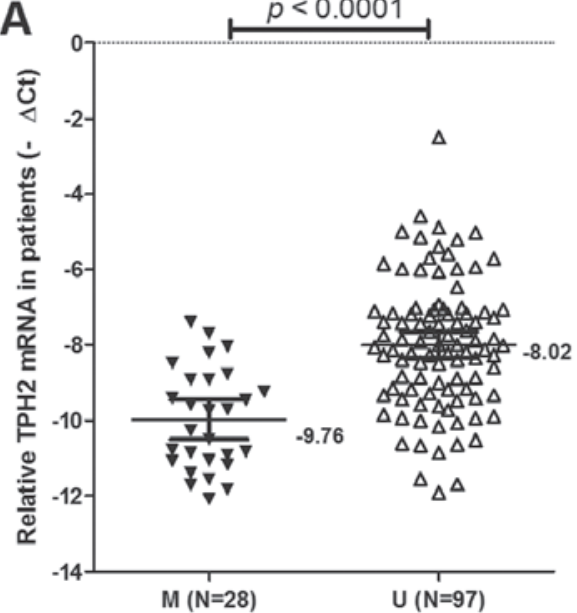

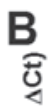

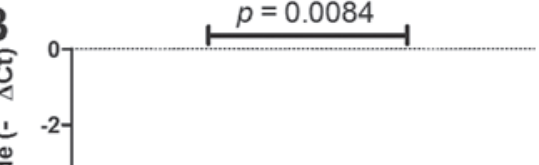

$\boldsymbol{\nabla}$

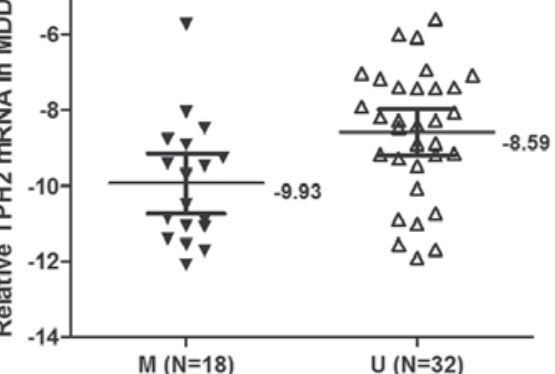

C

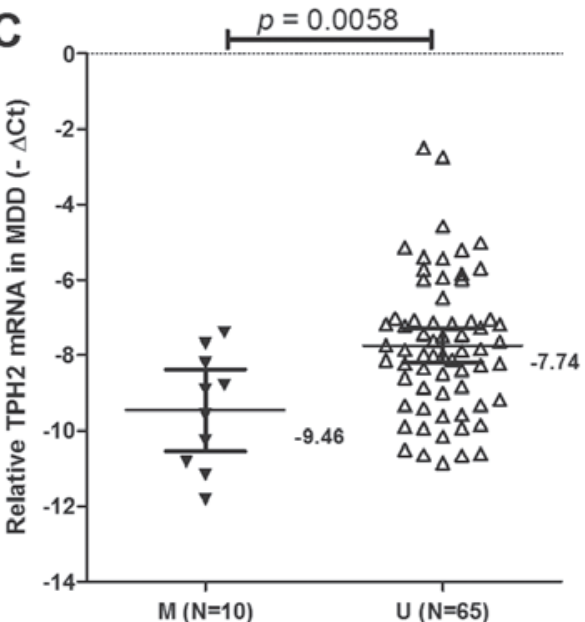

Figure 3. TPH2 mRNA expression and promoter methylation in patients with MDD. (A) TPH2 mRNA expression levels were lower in patients with methylated TPH2 promoters, as compared with those with unmethylated TPH 2 promoters. (B) TPH2 mRNA expression levels were lower in the MDD + suicide attempts group with methylated TPH2 promoters, as compared with those with unmethylated TPH2 promoters. (C) TPH2 mRNA expression levels were lower in the MDD control group with methylated TPH2 promoters, as compared with those with unmethylated TPH2 promoters. Statistical analyses were conducted using an unpaired t-test. $-\Delta \mathrm{Ct}$, - $\left(\mathrm{CT}_{\mathrm{TPH} 2}-\mathrm{CT}_{\beta \text {-actin }}\right) ; \mathrm{M}$, methylated; $\mathrm{U}$, unmethylated; $\mathrm{TPH} 2$, tryptophan hydroxylase-2; MDD, major depressive disorder; $\mathrm{CT}$, cycle threshold.

expression levels of TPH2, an RT-qPCR assay was conducted on samples from the MDD + suicide and MDD groups. The overall results are summarized in Fig. 1. TPH2 mRNA expression levels were lower in the MDD + suicide group [mean $-\Delta \mathrm{Ct} \pm$ standard error (SE),-9.07 \pm 0.24 ], as compared with the MDD group (mean $-\Delta \mathrm{Ct} \pm \mathrm{SE},-7.91 \pm 0.21$ ). The difference between TPH 2 expression levels in the MDD + suicide and MDD groups was significant $(\mathrm{P}=0.0005$; Fig. 1$)$. These results suggested that the downregulation of TPH2 gene expression may have an important role in the development of suicidal behaviors.

Methylation status of the TPH2 promoter in the MDD + suicide and MDD groups. The methylation status of the ТPH2 promoter region was analyzed in the MDD + suicide and MDD groups. The TPH2 promoter was methylated in $36.0 \%$ $(18 / 50)$ of MDD + suicide patients, as compared with in $13.0 \%$ (10/75) of MDD patients. Furthermore, the TPH2 promoter was unmethylated in $64.0 \%$ (32/50) of MDD + suicide patients, as compared with $87.0 \%(65 / 75)$ of MDD patients. The differences in TPH 2 methylation status between the MDD + suicide and MDD groups were statistically significant $(\mathrm{P}=0.0029$; Fig. 2).

Association of TPH2 methylation with TPH2 mRNA expression levels in the MDD + suicide and MDD groups. To determine whether TPH2 promoter methylation was correlated with the suppression of TPH2 mRNA expression in MDD, qPCR was used to detect the mRNA expression levels of TPH2 in all patients (Fig. 3). The mRNA expression levels of TPH2 were significantly decreased in the patients with TPH2 promoter methylation, as compared with those possessing an unmethylated TPH2 promoter (mean $-\Delta \mathrm{Ct} \pm \mathrm{SE},-9.76 \pm 0.25$ and $-8.02 \pm 0.17$, respectively; $\mathrm{P}<0.0001$; Fig. 3A).

In the MDD + suicide group, the mRNA expression levels of TPH2 were $-9.93 \pm 0.38$ in patients possessing a methylated $\mathrm{TPH} 2$ promoter and $-8.59 \pm 0.30$ in those with an unmethylated TPH2 promoter $(\mathrm{P}=0.0084$; Fig. $3 \mathrm{~B})$.
In the MDD group, the mRNA expression levels of TPH2 were $-9.46 \pm 0.48$ in the patients with a methylated TPH2 promoter, and $-7.74 \pm 0.23$ in those possessing an unmethylated TPH2 promoter ( $\mathrm{P}=0.0058$; Fig. $3 \mathrm{C})$. These results suggested that in patients with MDD exhibiting methylation of the TPH2 promoter, TPH 2 mRNA expression is likely to be downregulated.

Correlation of the methylation status of the TPH2 promoter with clinicopathological parameters in the $M D D+$ suicide and MDD groups. The methylation of specific gene promoters is considered a marker for various types of psychiatric disorder (33). The correlation of TPH2 methylation with the clinicopathological parameters of the MDD + suicide and MDD groups is shown in Table II. The methylation status of the TPH2 promoter was associated with depression, hopelessness and cognitive impairment $(\mathrm{P}=0.011,0.022$ and 0.015 , respectively) in the MDD + suicide group. In addition, methylation of TPH2 was only associated with depression $(\mathrm{P}=0.014)$ in the MDD group. Methylation of TPH2 was not associated with the remaining clinicopathological parameters evaluated, including gender, diurnal change, sleep disorders, anxiety and slow movement.

\section{Discussion}

Suicide has been identified as a serious public health problem, which is a result of an interplay between distal (e.g. genetic loading or family history of suicide) and proximal factors (e.g. presence of psychiatric disorders or events conferring acute stress) (1). Disorders of emotional regulation, including anxiety disorders and depression, are common and debilitating. Accumulating evidence has suggested an involvement of 5-HT in the regulation of emotion (34). The recent advent of TPH2 knockout mice, which lack the rate-limiting enzyme for 5-HT synthesis in the brain, has provided further insight into the brain serotonergic system and its role in emotional dysregulation (35). With increased understanding of the 
Table II. Methylation of the TPH2 gene promoter and clinical characteristics of patients with MDD.

\begin{tabular}{|c|c|c|c|c|c|c|}
\hline & \multicolumn{2}{|c|}{ MDD-suicide } & \multirow[b]{2}{*}{ P-value } & \multicolumn{2}{|c|}{ MDD } & \multirow[b]{2}{*}{ P-value } \\
\hline & $M(n=18)$ & $\mathrm{U}(\mathrm{n}=32)$ & & $M(n=10)$ & $\mathrm{U}(\mathrm{n}=65)$ & \\
\hline Gender & & & 0.869 & & & 0.649 \\
\hline Male & 8 & 15 & & 4 & 31 & \\
\hline Female & 10 & 17 & & 6 & 34 & \\
\hline Course of disease & & & 0.351 & & & 0.722 \\
\hline$<1$ year & 8 & 10 & & 4 & 29 & \\
\hline$\geq 1$ year & 10 & 22 & & 6 & 36 & \\
\hline Age at onset & & & 0.522 & & & 0.221 \\
\hline$<35$ years & 9 & 13 & & 3 & 33 & \\
\hline$\geq 35$ years & 9 & 19 & & 7 & 32 & \\
\hline Family history of mental illness & & & 0.584 & & & 0.961 \\
\hline Yes & 11 & 22 & & 7 & 45 & \\
\hline No & 7 & 10 & & 3 & 20 & \\
\hline Depression & & & 0.011 & & & 0.014 \\
\hline HDRS $<35$ & 4 & 19 & & 2 & 40 & \\
\hline $\mathrm{HDRS} \geq 35$ & 14 & 13 & & 8 & 25 & \\
\hline Hopelessness & & & 0.022 & & & 0.488 \\
\hline HDRS $<6$ & 7 & 23 & & 5 & 40 & \\
\hline $\mathrm{HDRS} \geq 6$ & 11 & 9 & & 5 & 25 & \\
\hline Cognitive impairment & & & 0.015 & & & 0.101 \\
\hline HDRS $<12$ & 6 & 22 & & 2 & 31 & \\
\hline $\mathrm{HDRS} \geq 12$ & 12 & 10 & & 8 & 34 & \\
\hline Diurnal change & & & 0.941 & & & 0.716 \\
\hline HDRS $<1$ & 6 & 11 & & 4 & 30 & \\
\hline $\mathrm{HDRS} \geq 1$ & 12 & 21 & & 6 & 35 & \\
\hline Sleep disorders & & & 0.053 & & & 0.488 \\
\hline HDRS $<2$ & 5 & 18 & & 3 & 27 & \\
\hline HDRS $\geq 2$ & 13 & 14 & & 7 & 38 & \\
\hline Anxiety & & & 0.352 & & & 0.855 \\
\hline HAMA $<21$ & 6 & 15 & & 4 & 28 & \\
\hline HAMA $\geq 21$ & 12 & 17 & & 6 & 37 & \\
\hline Slow movement & & & 0.309 & & & 0.820 \\
\hline HDRS $<8$ & 8 & 19 & & 5 & 35 & \\
\hline HDRS $\geq 8$ & 10 & 13 & & 5 & 30 & \\
\hline
\end{tabular}

HDRS, Hamilton Depression Rating Scale; HAMA, Hamilton Anxiety Rating Scale; TPH2, tryptophan hydroxylase-2; MDD, major depressive disorder; M, methylated; U, unmethylated. Statistical analyses were conducted using the Pearson $\chi^{2}$ and Fisher exact tests.

regulation of TPH2 activity, TPH2 may be used as a target for the development of novel treatments or for optimization of current therapies, which are expected to markedly improve the prevention and treatment of depression disorders (36).

TPH2 synthesizes neuronal 5-HT and its genetic variance is associated with numerous behavioral traits and psychiatric disorders. An association has been identified between TPH2 and genetically defined behavioral variations, and TPH 2 genetic variation may be used to predict the risk of affective disorders and the sensitivity to anti-depressant therapeutics $(5,37)$. TPH2 gene expression is tissue-specific and responsive to stressors, including adverse experiences during early life and adulthood (9). These findings suggested that epigenetic mechanisms may have important roles in the regulation of TPH2 expression (38). Epigenetic modifications, such as DNA methylation, are able to induce lasting and stable changes in gene expression, and have therefore been implicated in promoting the adaptive behavioral and neuronal changes that accompany depression (39).

The present study aimed to compare the gene expression levels of TPH 2 between patients with MDD who had attempted suicide and those who had not (controls), and to identify epigenetic methylation involved in TPH 2 regulation. The 
results of the present study demonstrated that the frequency of TPH 2 methylation was $36.0 \%$ in the MDD + suicide group, as compared with $13.0 \%$ in the MDD group. The mRNA expression levels of TPH2 were significantly decreased in the patients with methylated TPH 2 promoters, as compared with those with unmethylated TPH 2 promoters. These results suggested that methylation of the TPH2 promoter may silence TPH 2 mRNA expression in patients with MDD. Furthermore, a significant correlation was shown between the methylation status of the TPH 2 promoter and depression, hopelessness and cognitive impairment in the MDD + suicide group. The results of the present study demonstrated that TPH2 expression may be regulated by DNA methylation in the promoter region in patients with MDD.

Epigenetic modification of gene expression provides a mechanism for understanding the link between long-term effects of adverse life events and the alterations in gene expression that are associated with depression. Although epigenetics remain a developing field, future studies on epigenetic modifications of gene expression may provide novel biomarkers to predict future susceptibility and/or onset of MDD, improve diagnosis and aid in the development of epigenetics-based therapies for depression (23). TPH2 transcription and protein expression are modulated by neuronal differentiation in the central nervous system-originated cell line A1 mes-c-myc, which endogenously expresses TPH2, and promoter activity strongly increases with cell differentiation upon mutation of the neuron-restrictive silencer factor (NRSF)/RE1-silencing transcription factor-responsive element (40). The upstream segment of the TPH2 5'-UTR contains a binding motif for NRSF, which mediates transcriptional repression. Therefore, it is likely that the 5'-UTR serves as an 'on-off' switch for the regulation of TPH 2 expression (41).

In conclusion, the results of the present study provided novel insight into the epigenetic mechanisms of MDD. Increasing knowledge regarding the regulation of TPH2 expression will not only improve the understanding of 5-HT-stress interaction and the pathophysiology of neuropsychiatric disorders, but may also provide novel strategies for the treatment of stress-associated diseases.

\section{Acknowledgements}

The present study was supported by the Science and Technology Planning Project of Wuxi Municipality, China (grant no. CSE01N1118), the Medical Technology Project of Wuxi Hospital Management Center (grant no. YGZX1116), the Natural Science Foundation of China (grant no. 81372212), the Jiangsu Provincial Special Program of Medical Science (grant no. BL2013012) and the Health Talents Project for Jiangsu, China (grant nos. LJ201157 and RC2011038).

\section{References}

1. Hawton K and van Heeringen K: Suicide. Lancet 373: 1372-1381, 2009.

2. McGirr A, Renaud J, Séguin M, Alda M and Turecki G: Course of major depressive disorder and suicide outcome: A psychological autopsy study. J Clin Psychiatry 69: 966-970, 2008.

3. Cavanagh JT, Carson AJ, Sharpe M and Lawrie SM: Psychological autopsy studies of suicide: A systematic review. Psychol Med 33: 395-405, 2003
4. Pompili M, Innamorati M, Raja M, et al: Suicide risk in depression and bipolar disorder: Do impulsiveness-aggressiveness and pharmacotherapy predict suicidal intent? Neuropsychiatr Dis Treat 4: 247-255, 2008

5. Matthes S, Mosienko V, Bashammakh S, Alenina N and Bader M: Tryptophan hydroxylase as novel target for the treatment of depressive disorders. Pharmacology 85: 95-109, 2010.

6. Chen GL and Miller GM: Advances in tryptophan hydroxylase-2 gene expression regulation: New insights into serotonin-stress interaction and clinical implications. Am J Med Genet B Neuropsychiatr Genet 159B: 152-171, 2012.

7. Must A, Tasa G, Lang A, et al: Variation in tryptophan hydroxylase-2 gene is not associated to male completed suicide in Estonian population. Neurosci Lett 453: 112-114, 2009.

8. Tsai SJ, Hong CJ, Liou YJ, et al: Tryptophan hydroxylase 2 gene is associated with major depression and antidepressant treatment response. Prog Neuropsychopharmacol Biol Psychiatry 33: 637-641, 2009.

9. Waider J, Araragi N, Gutknecht L and Lesch KP: Tryptophan hydroxylase-2 (TPH2) in disorders of cognitive control and emotion regulation: A perspective.Psychoneuroendocrinology 36 : 393-405, 2011.

10. Del'Guidice T, Lemay F, Lemasson M, et al: Stimulation of 5-HT2C receptors improves cognitive deficits induced by human tryptophan hydroxylase 2 loss of function mutation. Neuropsychopharmacology 39: 1125-1134, 2014.

11. Carkaci-Salli N, Salli U, Tekin I, et al: Functional characterization of the S41Y (C2755A) polymorphism of tryptophan hydroxylase 2. J Neurochem 130: 748-758, 2014.

12. Arango V, Huang YY, Underwood MD and Mann JJ: Genetics of the serotonergic system in suicidal behavior. J Psychiatr Res 37: 375-386, 2003.

13. Gutknecht L, Jacob C, Strobel A, et al: Tryptophan hydroxylase-2 gene variation influences personality traits and disorders related to emotional dysregulation. Int J Neuropsychopharmacol 10: 309-320, 2007.

14. Lim JE, Pinsonneault J, Sadee W and Saffen D: Tryptophan hydroxylase 2 (TPH2) haplotypes predict levels of TPH2 mRNA expression in human pons. Mol Psychiatry 12: 491-501, 2007.

15. Chen GL, Vallender EJ and Miller GM: Functional characterization of the human TPH2 5' regulatory region: Untranslated region and polymorphisms modulate gene expression in vitro. Hum Genet 122: 645-657, 2008.

16. Chen GL and Miller GM: Rhesus monkey tryptophan hydroxylase-2 coding region haplotypes affect mRNA stability. Neuroscience 155: 485-491, 2008.

17. Roche S and McKeon P: Support for tryptophan hydroxylase-2 as a susceptibility gene for bipolar affective disorder. Psychiatr Genet 19: 142-146, 2009.

18. Zhang C, Li Z, Shao Y, et al: Association study of tryptophan hydroxylase-2 gene in schizophrenia and its clinical features in Chinese Han population. J Mol Neurosci 43: 406-411, 2011.

19. Zhang Y, Zhang C, Yuan G et al: Effect of tryptophan hydroxylase-2 rs7305115SNP on suicide attempts risk in major depression. Behav Brain Funct 6: 49, 2010.

20. Perroud N, Neidhart E, Petit B, et al: Simultaneous analysis of serotonin transporter, tryptophan hydroxylase 1 and 2 gene expression in the ventral prefrontal cortex of suicide victims. Am J Med Genet B Neuropsychiatr Genet 153B: 909-918, 2010.

21. De Luca V, Likhodi O, Van Tol HH, Kennedy JL and Wong AH: Gene expression of tryptophan hydroxylase 2 in post-mortem brain of suicide subjects. Int J Neuropsychopharmacol 9: 21-25, 2006.

22. Domschke K, Tidow N, Schwarte K, et al: Serotonin transporter gene hypomethylation predicts impaired antidepressant treatment response. Int J Neuropsychopharmacol 17: 1167-1176, 2014.

23. Dalton VS, Kolshus E and McLoughlin DM: Epigenetics and depression: Return of the repressed. J Affect Disord 155: $1-12,2014$.

24. Klengel T, Pape J, Binder EB and Mehta D: The role of DNA methylation in stress-related psychiatric disorders. Neuropharmacology 80: 115-132, 2014.

25. Williams JB: A structured interview guide for the Hamilton Depression Rating Scale. Arch Gen Psychiatry 45: 742-747, 1988.

26. Leung CM, Wing YK, Kwong PK, Lo A and Shum K: Validation of the Chinese-Cantonese version of the hospital anxiety and depression scale and comparison with the Hamilton Rating Scale of Depression. Acta Psychiatr Scand 100: 456-461, 1999.

27. Baca-Garcia E, Perez-Rodriguez MM, Basurte-Villamor I, et al: Diagnostic stability of psychiatric disorders in clinical practice. Br J Psychiatry 190: 210-216, 2007. 
28. Spitzer RL and Wakefield JC: DSM-IV diagnostic criterion for clinical significance: Does it help solve the false positives problem? Am J Psychiatry 156: 1856-1864, 1999.

29. Hamilton M. The assessment of anxiety states by rating. Br J Med Psychol 32: 50-55, 1959.

30. Ling Y, Zhang C, Xu Y, et al: Promoter methylation-associated silencing of p27kip1 gene with metastasis in esophageal squamous cell carcinoma. Mol Med Rep 9: 1075-1079, 2014.

31. Li LC and Dahiya R: MethPrimer: Designing primers for methylation PCRs. Bioinformatics 18: 1427-1431, 2002.

32. Livak KJ and Schmittgen TD: Analysis of relative gene expression data using real-time quantitative PCR and the 2(-Delta Delta C(T)) method. Methods 25: 402-408, 2001.

33. Aberg KA, McClay JL, Nerella S, et al: Methylome-wide association study of schizophrenia: identifying blood biomarker signatures of environmental insults. JAMA Psychiatry 71: 255-264, 2014.

34. Meneses A and Liy-Salmeron G: Serotonin and emotion, learning and memory. Rev Neurosci 23: 543-553, 2012.

35. Araragi N and Lesch KP: Serotonin (5-HT) in the regulation of depression-related emotionality: Insight from 5-HT transporter and tryptophan hydroxylase-2 knockout mouse models. Curr Drug Targets 14: 549-570, 2013.
36. Chen GL and Miller GM: Tryptophan hydroxylase-2: An emerging therapeutic target for stress disorders. Biochem Pharmacol 85: 1227-1233, 2013.

37. Popova NK and Kulikov AV: Targeting tryptophan hydroxylase 2 in affective disorder. Expert Opin Ther Targets 14: 1259-1271, 2010.

38. Gardner KL, Hale MW, Oldfield S, Lightman SL, Plotsky PM and Lowry CA: Adverse experience during early life and adulthood interact to elevate tph2 mRNA expression in serotonergic neurons within the dorsal raphe nucleus. Neuroscience 163: 991-1001, 2009.

39. Mahgoub M and Monteggia LM: Epigenetics and psychiatry. Neurotherapeutics 10: 734-741, 2013.

40. Gentile MT, Nawa Y, Lunardi G, Florio T, Matsui H and Colucci-D'Amato L: Tryptophan hydroxylase 2 (TPH2) in a neuronal cell line: Modulation by cell differentiation and NRSF/rest activity. J Neurochem 123: 963-970, 2012.

41. Patel PD, Bochar DA, Turner DL, Meng F, Mueller HM and Pontrello CG: Regulation of tryptophan hydroxylase-2 gene expression by a bipartite RE-1 silencer of transcription/neuron restrictive silencing factor (REST/NRSF) binding motif. J Biol Chem 282: 26717-26724, 2007. 